\title{
Hirayama disease in a 17-year-old Chinese man
}

Jacqueline Ching Man Sitt ${ }^{1}$, MBBS, Eva Lai Wah Fung ${ }^{2}$, FHKCPaed, FHKAM, Edmund Hok-Yuen $\underline{\text { Yuen }}^{1}$, FRCR, FHKAM, Anil Tejbhan $\underline{\text { Ahuja }}^{1}$, FRCR, FHKAM

\begin{abstract}
Hirayama disease is an uncommon cervical myelopathy associated with neck flexion. It has been postulated to be related to the anterior shifting of the posterior dura of the lower cervical dural canal during neck flexion, resulting in lower cervical cord atrophy with asymmetric flattening. We report a case of Hirayama disease in a 17-year-old Chinese man and demonstrate the use of dynamic flexion magnetic resonance imaging of the cervical spine in the diagnosis of the disease.
\end{abstract}

Keywords: cervical spine, flexion magnetic resonance imaging, Hirayama disease

\section{INTRODUCTION}

Hirayama disease is an uncommon cervical myelopathy associated with neck flexion. Typically presenting in adolescent boys and young men, it is postulated to be related to the anterior shifting of the posterior dura of the lower cervical dural canal during neck flexion, resulting in lower cervical cord atrophy with asymmetric flattening. ${ }^{(1)}$ A high awareness of its natural history, careful physical examination, and the use of neurophysiological studies and dynamic magnetic resonance (MR) imaging studies, especially the flexion view of the cervical spine, provide key information necessary for making an accurate diagnosis in patients with Hirayama disease.

\section{CASE REPORT}

A 17-year-old Chinese man with no prior medical history presented to the paediatric neurology unit at the Prince of Wales Hospital, Hong Kong, with a two-year history of progressive weakness and numbness of the hands. The weakness initially started in the left hand, which progressively increased in severity and extended to involve the right hand. The numbness was provoked by flexion of the neck, and symptoms resolved upon returning the neck to the neutral or extended position. The patient also noted asymmetry in the size of his hands, with muscle wasting more severely on the left side. There was no history of trauma.

Physical examination revealed marked atrophy of the thenar, hypothenar and interosseous muscles of both hands, but more severely affecting the left side (Fig. 1a). Mild atrophic changes of the muscles of both forearms were also noted, although the brachioradiales were spared. Full abduction, adduction of the digits, opposition of the thumbs and palmar grasps were impaired (Fig. 1b). Overall, the function of the left hand was more severely affected. Deep tendon reflexes and sensation were preserved. The power of the proximal muscles of both upper limbs was normal. The lower limbs were unremarkable and cranial nerve functions were intact.

Neurophysiological studies revealed active denervation changes to the small muscles of the hands while motor and sensory nerve conduction studies to the bilateral median and ulnar nerves were within normal limits. Findings pointed toward
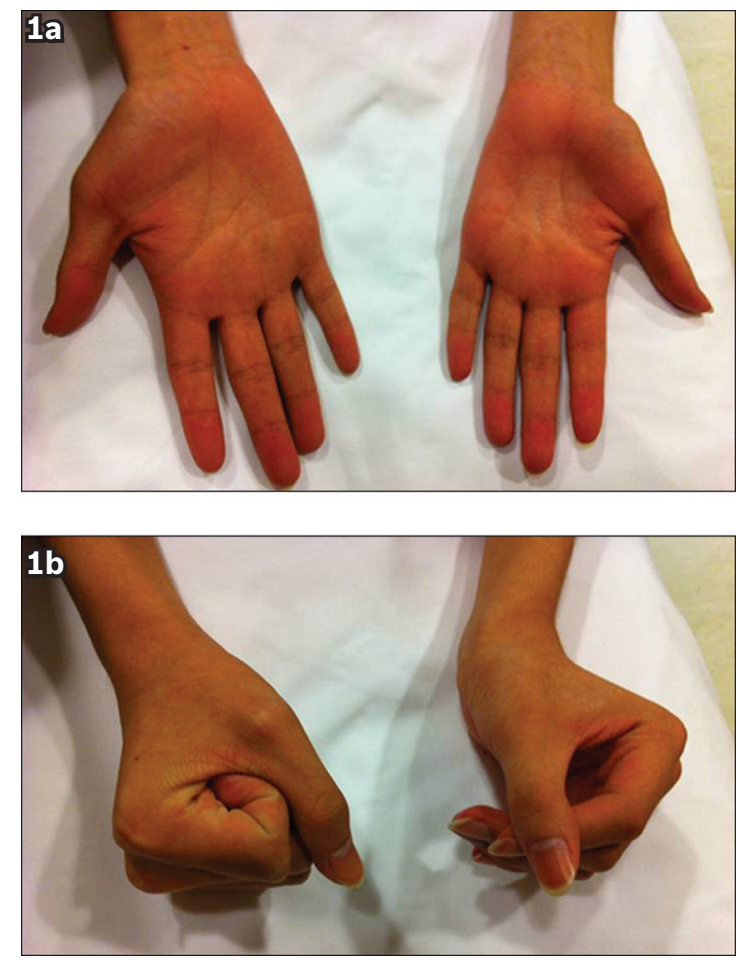

Fig. 1 Photographs show (a) marked atrophy of the thenar, hypothenar and interosseous muscles of both hands, but more severely affecting the left side, and (b) impairment of the palmar grasp.

disorder of the anterior horn cells of the lower cervical levels, and a diagnosis of Hirayama disease was suspected.

Plain radiographs of the cervical spine showed loss of normal lordosis, but were otherwise unremarkable. Non-flexion cervical T2-weighted MR imaging showed cord atrophy at the C5-C6 level, predominantly affecting the bilateral anterior aspects, and that the posterior dura was detached (Fig. 2). The anterior thecal sac was capacious on sagittal extension view on MR imaging. As the diagnosis of Hirayama disease was raised based on the overall clinical findings, an additional sagittal flexion view on MR imaging was performed. Upon flexion of the neck, anterior displacement of the posterior wall of the cervical dural canal from levels C4 to T3 was noted, with marked flattening of the cord 
and complete effacement of the anterior thecal sac (Figs. 3a \& b). Postcontrast flexion MR imaging showed a homogeneously contrast-enhancing posterior epidural mass at these levels, with multiple small signal voids noted within (Fig. 4a). This mass disappeared when the neck returned to the non-flexion position, and represented the engorged posterior epidural venous plexus due to dural shifting (Fig. 4b).

Overall, the clinical and imaging features were compatible with the diagnosis of Hirayama disease. A neck collar was given to the patient to prevent neck flexion.

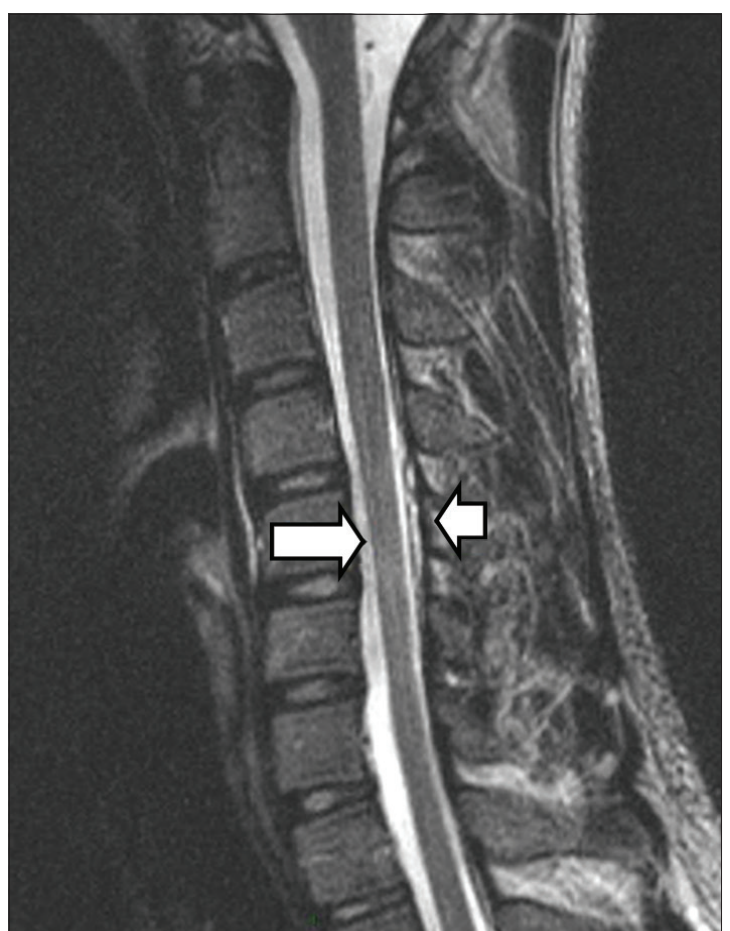

Fig. 2 Non-flexion cervical T2-W sagittal MR image shows cord atrophy at the C5-C6 level (arrow), predominantly affecting the bilateral anterior aspects, and the posterior dura to be detached (arrowhead).

\section{DISCUSSION}

Hirayama disease is a benign cervical myelopathy associated with neck flexion. ${ }^{(1)}$ First described in 1959 by Hirayama et al, (2) the disease has also been described in the literature as juvenile muscular atrophy of the distal upper extremity, ${ }^{(3)}$ juvenile muscular atrophy of a unilateral upper extremity, ${ }^{(2)}$ juvenile asymmetric segmental spinal muscular atrophy, ${ }^{(4)}$ benign focal amyotrophy or monomelic amyotrophy. ${ }^{(3,5)}$

Typically, adolescent boys and young men in their 20s and 30 s are affected, and patients present with the insidious onset of muscular weakness, numbness and atrophy of the hands and forearms. Symptoms are slowly progressive and often reported to be worsened by cold weather. The brachioradialis is usually spared. (1) Different patterns of involvement have been reported, with unilateral involvement being most commonly reported, followed by asymmetric and symmetric bilateral involvement. ${ }^{(6)}$ There is no sensory or pyramidal tract involvement. ${ }^{(7)}$

The exact pathogenesis of the disease is still uncertain. However, it is generally believed to be associated with a tight dural canal during flexion of the neck. ${ }^{(1,8)}$ The relatively short and tight dura mater seen in patients with Hirayama disease is unable to compensate for the increased length of the vertebral canal during neck flexion, thus resulting in the dural canal tightening up during neck flexion, which leads to an anterior shift of the posterior dural wall, leading to spinal cord compression. ${ }^{(8)}$ Repeated neck flexion results in multiple episodes of ischaemia and chronic trauma to the spinal cord, which eventually leads to myelopathy, as evidenced by asymmetric lower cervical cord thinning. ${ }^{(1,9)}$ A pathological study by Hirayama et al demonstrated lesions in the anterior horns of the spinal cord from the lower cervical to upper thoracic levels, and particularly at the C7 and C8 levels. ${ }^{(10)}$

Hirayama disease is uncommon and is therefore easily overlooked or misdiagnosed. A high awareness of its natural history, careful physical examination and detailed

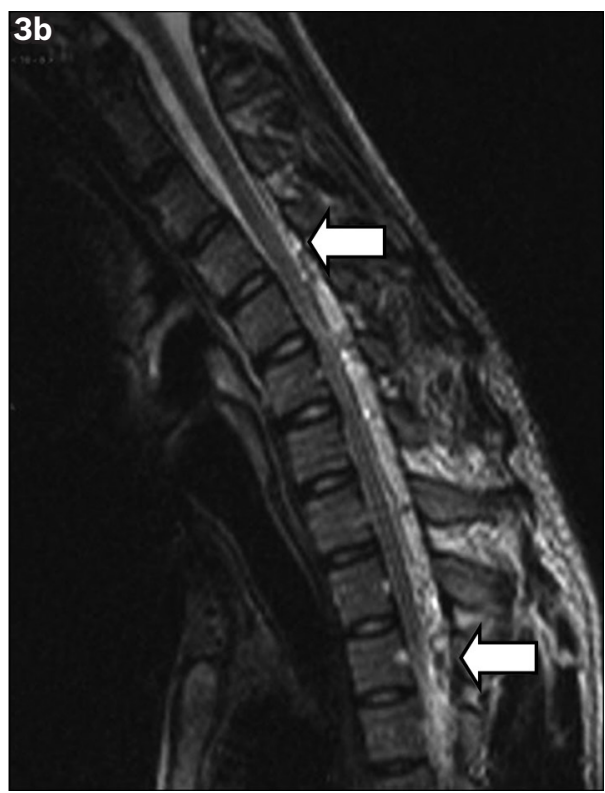

Fig. 3 Flexion (a) T1-W and (b) T2-W sagittal MR images show anterior displacement of the posterior wall of the cervical dural canal from levels C4 to T3 (arrows), with flattening of the cord and complete effacement of the anterior thecal sac. 

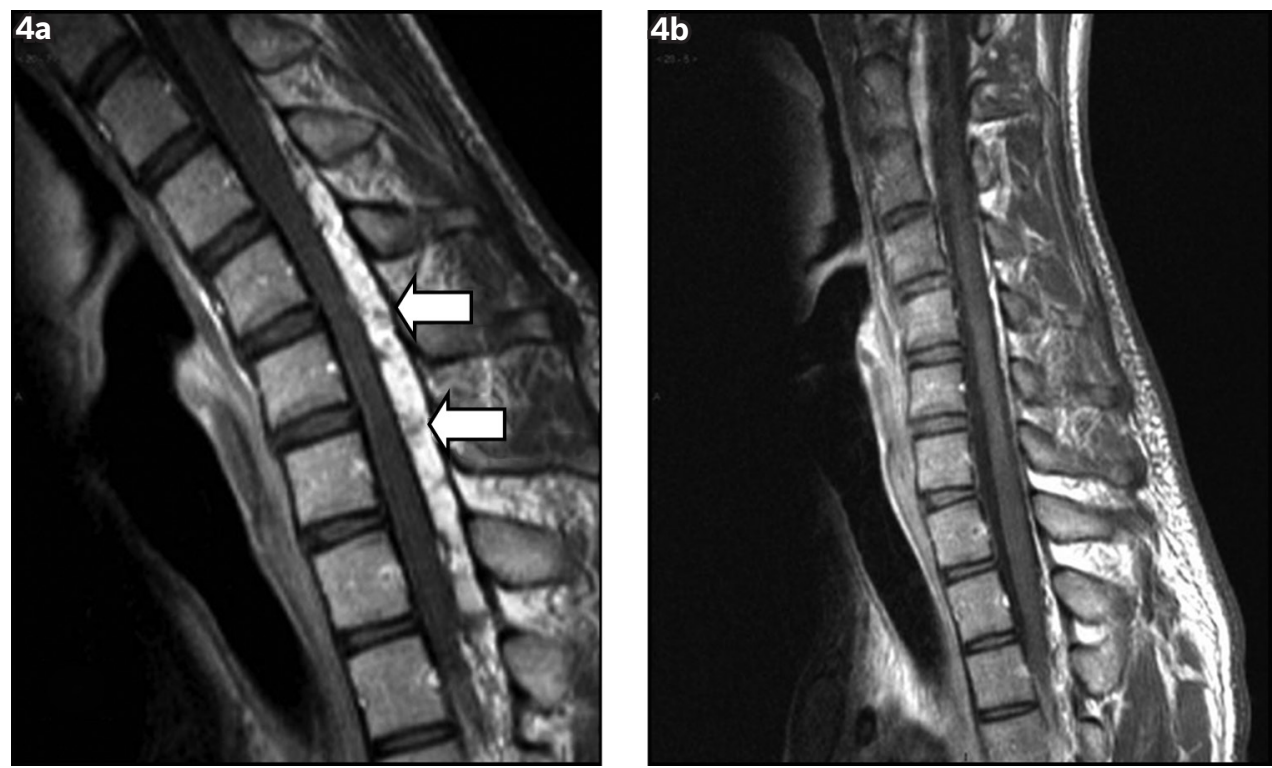

Fig. 4 Dynamic T1-W postcontrast sagittal flexion MR images show (a) a homogeneously contrast-enhancing posterior epidural mass with multiple small signal voids within (arrows); and (b) disappearance of the epidural mass when the neck returns to a neutral position.

neurophysiological studies all have a role to play in narrowing down the list of differential diagnoses. As in our patient, imaging findings, particularly of dynamic MR imaging studies, were key in confirming the diagnosis of Hirayama disease. ${ }^{(11)}$

Cervical spine radiographs are nonspecific, with no focal abnormalities demonstrated except for straight alignment. ${ }^{(12)}$ In contrast, MR imaging studies of the cervical spine in a neutral position can reveal several features such as localised lower cervical cord atrophy, asymmetrical cord flattening, loss of attachment between the posterior dural sac and subjacent lamina, as well as non-compressed intramedullary high $\mathrm{T} 2$ signal intensity. Among these, the loss of attachment of the posterior dura is a most valuable finding for the diagnosis of Hirayama disease. ${ }^{(13)} \mathrm{MR}$ imaging of neck flexion shows the anterior displacement of the posterior dura as well as a homogeneously contrast-enhancing, crescent-shaped mass in the posterior epidural space of the lower cervical canal. This mass typically disappears when the neck returns to a neutral position, confirming it to be a congested posterior internal vertebral venous plexus rather than a vascular malformation. ${ }^{(1,8,13)}$

For patients with Hirayama disease, avoidance of neck flexion by using neck collars remains the first-line treatment, which is also effective in stopping the progression of the disease. ${ }^{(9)}$ Surgical interventions such as cervical decompression and fusion have also shown some promising results ${ }^{(11)}$ and may be considered for selective patients. It should be noted that the anterior decompressive approach may be better for patients with anterior effacement and severe cervical kyphosis on MR imaging during neck flexion. ${ }^{(11)}$ Early diagnosis of the disease is of the essence for patients with Hirayama disease, so that early treatment can be instituted.

In summary, Hirayama disease should always be considered in a young patient presenting with hand and forearm muscle atrophy, and asymmetrical thinning of the cervical spinal cord. Dynamic flexion MR imaging studies of the cervical spine are key for its accurate diagnosis, as the forward migration of the posterior surface of the dura mater, with compression of the anterior aspect of the spinal cord, can be demonstrated using this modality. ${ }^{(9)}$

\section{REFERENCES}

1. Chen CJ, Chen $C M, W u ~ C L$, et al. Hirayama disease: $M R$ diagnosis. AJNR Am J Neuroradiol 1998; 19:365-8.

2. Hirayama K, Toyokura Y, Tsubaki T. Juvenile muscular atrophy of unilateral upper extremity: a new clinical entity. Psychiatr Neurol Jpn 1959; 61:2190-7.

3. Biondi A, Dormont D, Weitzner I Jr, et al. MR Imaging of the cervical cord in juvenile amyotrophy of distal upper extremity. AJNR Am J Neuroradiol 1989; 10:263-8.

4. Pradhan S, Gupta RK. Magnetic resonance imaging in juvenile asymmetric segmental spinal muscular atrophy. J Neurol Sci 1997; 146:133-8.

5. Kwon O, Kim M, Lee KW. A Korean case of juvenile muscular atrophy of distal upper extremity (Hirayama disease) with dynamic cervical cord compression. J Korean Med Sci 2004; 9:768-71.

6. Hirayama K, Tokumaru Y. Cervical dural sac and spinal cord in juvenile muscular atrophy of distal upper extremity. Neurology 2000; 54:1922-6.

7. Sobue I, Saito N, lida M, Ando K. Juvenile type of distal and segmental muscular atrophy of upper extremities. Ann Neurol 1978; 3:429-32.

8. Kikuchi S, Tashiro K, Kitagawa K, Iwasaki Y, Abe H. [A mechanism of juvenile muscular atrophy localized in the hand and forearm (Hirayama's disease): flexion myelopathy with tight dural canal in flexion]. Rinsho Shinkeigaku 1987; 27:412-9. Japanese.

9. Gandhi D, Goyal M, Bourque PR, Jain R. Case 68: Hirayama disease. Radiology 2004; 230:692-6.

10. Hirayama K, Tomonaga M, Kitano K, et al. Focal cervical poliopathy causing juvenile muscular atrophy of distal upper extremity: a pathological study. J Neurol Neurosurg Psychiatry 1987; 50:285-90.

11. Lin MS, Kung WM, Chiu WT, et al. Hirayama disease. J Neurosurg Spine 2010; 12:629-34

12. Mukai E, Sobue I, Muto T, Takahashi A, Goto S. [Abnormal radiological findings on juvenile-type distal and segmental muscular atrophy of upper extremities]. Rinsho Shinkeigaku 1985; 25:620-6. Japanese.

13. Chen CJ, Hsu HL, Tseng YC, et al. Hirayama flexion myelopathy: neutralposition MR imaging findings--importance of loss of attachment. Radiology $2004 ; 231: 39-44$ 